\title{
A Design and Analysis Methodology for Component-Based Real-Time Architectures of Autonomous Systems
}

\author{
Gobillot Nicolas ${ }^{1, *}$, Lesire Charles ${ }^{2}$, Doose David ${ }^{2}$ \\ 1 Ifremer, La Seyne-sur-Mer, France \\ 2 ONERA, Toulouse, France \\ * Corresponding author : Nicolas Gobillot, email address : nicolas.gobillot@ifremer.fr
}

\begin{abstract}
:
The integration of autonomous robots in real applications is a challenge. It needs that the behaviour of these robots is proved to be safe. In this paper, we focus on the real-time software embedded on the robot, and that supports the execution of safe and autonomous behaviours. We propose a methodology that goes from the design of component-based software architectures using a Domain Specific Language, to the analysis of the real-time constraints that arise when considering the safety of software applications. This methodology is supported by a code generation toolchain that ensures that the code eventually executed on the robot is consistent with the analysis performed. This methodology is applied on a ground robot exploring an area. Categories (2), (3)
\end{abstract}

Keywords : Robotics software, Real-time analysis, Modelling language 


\section{Introduction}

Nowadays dangerous, repetitive or precision requiring jobs are done by robots like flying drones, industrial assembly arms or medical assistants. In all these cases, human beings can interact with the machines. It is therefore essential to guarantee that every part of the robot software and hardware will produce a safe behaviour. For instance, we need to ensure that these robots will not damage themselves or their environment and more importantly that they do not hurt any human-being. Safety concern has already been considered regarding several aspects of robotics: collision avoidance $[1$, 
$2,3]$, human awareness [4,5], fault detection [6,7], or controller synthesis [8,9]. In this paper we are concerned with the analysis of the fulfilment of real-time constraints on the robot software. Real-time constraints are part of the non-functional requirements that arise when guaranteeing the safety of a critical software. Real-time constraints are of course not sufficient, as the functionnal part of the architecture must also be proved. Violating real-time constraints can however lead to inconsistent or unsafe behaviours of the functional part of the software architecture. For instance, $[10,11]$ have analysed the impact of software delays in control systems regarding the system stability. Verifying real-time constraints of embedded control architectures is therefore necessary to guarantee a safe behavior of the robotic system.

\subsection{Real-time analysis}

Real-time guarantees are brought by timing analyses on the software. A real-time analysis is based on the computation of the Worst Case Response Time (WCRT) of tasks [12]. The WCRT of a task represents the longest time between its activation and the end of its execution, including all the possible interruptions. During the past decades, the widely known Liu and Layland [13] fixed priority models, as well as dynamic priority task models such as Earliest Deadline First (EDF, [14]) have been precise enough for software analysis. Such models use a common and simple task model (see Fig. 1): each task $\tau_{i}$ is defined by a period $T_{i}$ and a deadline $D_{i}$. At each period, corresponding to a release date $r_{k}$, the task instance has to be executed. The execution, that can possibly be preempted, takes a time $C_{k}$ (in the figure, $C_{k}=C_{k}^{(1)}+C_{k}^{(2)}$ ). In classical task model, this computation time is noted $C_{i}$ and is represented by an upper bound of its value, the Worst Case Execution Time (WCET). The response time $R_{k}$ is then the delay between $r_{k}$ and the end of execution, and we have to prove that $R_{k}<D_{i}, \forall i, k$. Due to the increasing complexity of the real-time systems, the

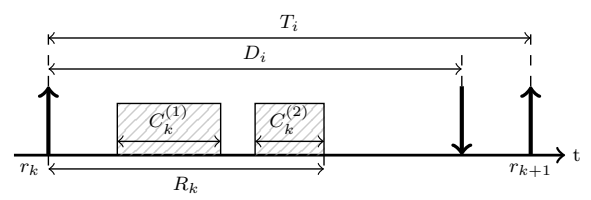

Fig. 1: Task model illustration: $k$-th execution of $i$-th task $\tau_{i}$.

analysis methods had to be adapted taking into account resource partitioning $[15,16$, 17]. Other improvements needed to account for task interdependency [18]. All these approaches for real-time analysis of software applications are based on a model of the software. It is then very important to have an execution model which is as close as possible to the practical execution that takes place on robots.

\subsection{Software design in robotics}

Developing autonomous robots has lead researchers to design several concepts to organise functional parts of the software architecture, including, among others, sensing, planning, and acting functions. A classical architecture concept is the 3-level 
architecture [19], and other paradigms have been proposed in the past decades [20,21, $22,23]$. Such developments are the application part of the software architecture: they concern the implementation of the functions embedded on the robot, but they must also settle on a middleware and/or operating system in order to be eventually deployed. Moreover, the development and implementation of these applications encounter some specificity when developing robotic application. They need for fast evolutions that lead to short development cycles of several month unlike in aeronautics or in the nuclear field which have development cycles of tenth of years. Furthermore the complexity of embedded systems software architectures increases with more and more tasks involved, making the use of software design methodologies and tools mandatory. To help the software robot developer, some recent design approaches used in robotics are based both on a middleware to help the development, and on a component-based approach to help managing the development cycle. Such approaches are typically relevant when the system under study is a critical embedded system (or at least some part of the software is critical). A component-based design pattern allows the software architect to build a robotic architecture by assembling existing software components [24,25]. Table 1 summarizes several approaches regarding either middlewares for developing robotic applications (top rows) and model-based processes (bottom rows).

Table 1: Summary of related references and their respective features $(\checkmark$ when supported, $\sim$ when partially supported or limited, $\boldsymbol{X}$ when unsupported).

\begin{tabular}{|c|c|c|c|c|c|c|c|c|}
\hline & & 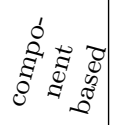 & 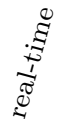 & $\overrightarrow{0}$ & 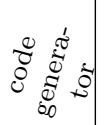 & 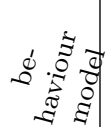 & $\begin{array}{l}E \\
0 \\
0 \\
0\end{array}$ & $\frac{E}{\delta}$ \\
\hline \multirow{5}{*}{ 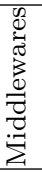 } & cisst [26] & $\sqrt{ }$ & $\sqrt{ }$ & $x$ & $x$ & $x$ & $x$ & $x$ \\
\hline & RTC [27] & $\sqrt{ }$ & $\checkmark$ & $x$ & $\sqrt{ }$ & $x$ & $x$ & $x$ \\
\hline & RoboComp $[28,29]$ & $\sqrt{ }$ & $\sqrt{ }$ & $\checkmark$ & $\sqrt{ }$ & $x$ & $x$ & $x$ \\
\hline & Orocos $[30]$ & $\sqrt{ }$ & $\sqrt{ }$ & $x$ & $x$ & $\sqrt{ }$ & $x$ & $x$ \\
\hline & $\operatorname{ROS}[31]$ & $\checkmark$ & $x$ & $x$ & $x$ & $x$ & $x$ & $x$ \\
\hline \multirow{6}{*}{ 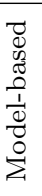 } & BIP [32] & $\sqrt{ }$ & $\sqrt{ }$ & $\sim$ & $\sqrt{ }$ & $\sqrt{ }$ & $x$ & $x$ \\
\hline & CPAL [33] & $\sqrt{ }$ & $\sqrt{ }$ & $\checkmark$ & $x$ & $\checkmark$ & $\sqrt{ }$ & $\checkmark$ \\
\hline & Rock $[34]$ & $\sqrt{ }$ & $\sqrt{ }[30]$ & $\sim$ & $\sqrt{ }$ & $\sqrt{ }$ & $x$ & $x$ \\
\hline & BRIDE [35] & $\checkmark$ & $\boldsymbol{X}_{[31]}$ & $\checkmark$ & $\checkmark$ & $\checkmark$ & $x$ & $x$ \\
\hline & $\mathrm{G}^{\mathrm{en}}{ }_{\mathrm{o}} \mathrm{M}[36]$ & $\checkmark$ & $\sim$ & $\checkmark$ & $\checkmark$ & $\checkmark$ & $\sim[37]$ & $\sim[37]$ \\
\hline & SmartSoft $[38,39]$ & $\sqrt{ }$ & $\sqrt{ }$ & $\checkmark$ & $\checkmark$ & $x$ & $x$ & $\sim[40]$ \\
\hline
\end{tabular}

Middlewares provide operating system and hardware abstractions. A middleware typically proposes an Application Programming Interface to develop and deploy tasks and threads without taking into account the operating system and thus the hardware specificities. While we can find several robotic-oriented middlewares with real-time capabilities [26,27,29,30], only Orocos-RTT [30] uses a behaviour model of components, based on a Finite State-Machine. Moreover, Orocos-RTT is widely used, in particular because of its integration with ROS [31]. Component-based approaches are often associated with modelling languages, such as Domain Specific Languages (DSL), that allow to develop software components in more abstract languages. Interesting approaches with respect to real-time and behaviour models are summarized in Tab. 1: BIP [32] and CPAL [33] have their own execution engine; BRIDE [35] and Rock [34] 
provide model-based design of components and allow to respectively generate ROS and Orocos components; $\mathrm{G}_{\mathrm{o}}^{\mathrm{en}} \mathrm{M}$ [36] proposes a rich component behaviour model, and can generate code into several middlewares (e.g., Orocos, ROS). Real-time evaluations in $\mathrm{G}^{\mathrm{en}}{ }_{\mathrm{o}} \mathrm{M}$ [37] are only based on raw measurements, and make the hypothesis that functions execution will not be preempted by the operating system. SmartSoft [38, 39] allows to generate real-time code based on the DDS framework and estimates the WCRT using Cheddar [40] without using the component behaviour model.

\subsection{Contribution}

The literature presented here above shows that there is no toolchain for real-time development of robotic software components that is complete, i.e. that provides a model-based development of real-time components, coupled with an accurate analysis of real-time properties. In this paper, we propose a design and real-time analysis process that is compliant with the current usage of robotic software developers, uses an accurate model of the resulting execution of the application, and provides relevant real-time analysis results that will help developpers to improve the overall safety of robotic systems. Compared to the works presented in Tab. 1, we have developed a model-based process that generates Orocos code, with accurate WCET/WCRT evaluations based on the component behaviour models. This process (Fig. 2) settles on:

- The design of architecture models using a Domain Specific Language (see Sect. 3);

- Code generation using state-of-the-art robotic middlewares (see Sect. 6);

- Architecture execution, along with an accurate execution model (see Sect. 4);

- A real-time analysis algorithm that computes accurate WCRTs (see Sect. 5).

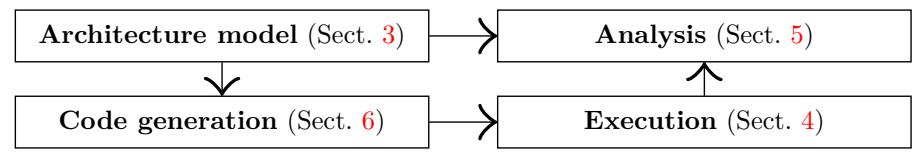

Fig. 2: Sketch of the proposed process.

Part of this work has already been published:

- a first version of the modelling language has been presented in [41]; here we present an updated version of the language with its complete grammar and concrete code snapshots used in an experiment illustrating the whole process;

- the real-time analysis presented in [42] is further detailed in this paper;

- the application case has been published in [43] with a focus on obtaining WCET from measures; this point is briefly described in this paper, but the application is further detailed, with code snapshots and more complete results.

\section{Case study}

The contribution presented in this paper has been applied to several case studies: a ground robot for exploration of indoor environments (Fig. 3a), a ground robot for 
patrolling missions to secure infrastructures (Fig. 3b), a ground robot for inspection of airport traffic lights (Fig. 3c), and a UAV performing a search-and-track mission [44].

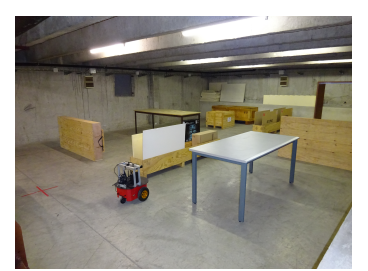

(a) Indoor exploration with a P3-DX robot

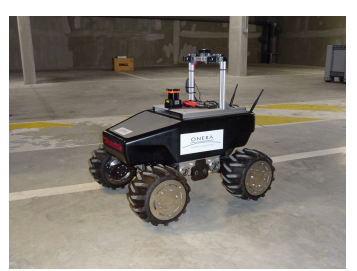

(b) Outdoor patrolling with a Summit XL robot

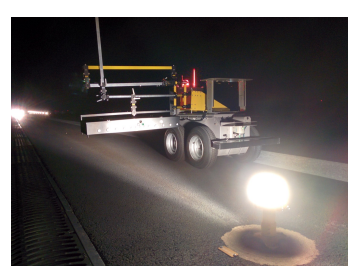

(c) Airport light inspection with a $4 \mathrm{MOB}$ robot

Fig. 3: Case studies

The architectures of the ground robots are very similar regarding the control components. In this paper we use the architecture of the Pioneer P3-DX robot used in the indoor exploration mission, as it provides interesting results that lead to the final discussions of this paper (Sect. 7.3). The Pioneer P3-DX robot is made of: (1) the mobile base with two motorized wheels to drive the robot and a passive caster wheel, (2) seven front-facing ultrasonic range finders and (3) an embedded low-level controller to drive the two motors and the ultrasonic sensors. On top of this, we added a high-level computer embedding a four core $1.83 \mathrm{GHz}$ processor, a laser scanner and a $3 \mathrm{D}$ camera. The high-level computer communicates with the proprietary low-level controller through its unique serial 9600bauds RS232 interface. In the context of our work, we configured the high-level computer to run a real-time patched Linux distribution.

\section{The MAUVE language}

As stated in the introduction, we need to catch best practices of robotics software development to build an accurate execution model of the resulting application. This is done by providing a modelling language to the developer. General purposes languages, such as AADL [45], UML [46] or MARTE [47] have been defined to model complex systems and used in several applications such that in the transportation or nuclear industry. These languages may be suited to have an abstract model of a complex robotic system $[48,49]$, but are limited when we need for an accurate model of execution. In robotics, and more generally in cyber-physical systems, a lot of works have defined and used DSLs. Contrary to general purpose languages, these DSLs are specific to some domains, then restricting their applicability, but have the good level of abstraction to model very deeply some parts of the system. Some of the works referenced in Tab. 1 have DSLs to model component-based architectures. However, none of these DSLs allows to model accurately component behaviours and their temporal execution, which are some mandatory elements to perform accurate realtime analyses. We then decided to define a new DSL, MAUVE. A first version of this DSL has been presented in [41]. This section presents an updated version of the model with better notations, particularly for the data ports access and parameter 
accessors for the codels. Moreover, we provide here the complete grammar of the MAUVE language, as it is a good way to highlight the several concepts of the language. However, note that the grammar itself is not sufficient to describe all the MAUVE model, as it also settles on complementary tools such that a type checker (to check the type of ports, components, ... ) and some semantic analysis rules (like avoiding multiple definitions of the same element). Moreover, to present MAUVE in a concise way, we have omitted some language primitives that could be trivially defined (like strings definition for type names and element ids).

\subsection{Codels declaration}

Codels are declarations of functions that may be used by the components (see Listing 1). Only the function prototype is defined at the model level, the function core being implemented in a target language. The term codel, as originally proposed by [36], stands for elementary code.

Listing 1: Part of the MAUVE grammar defining codels.

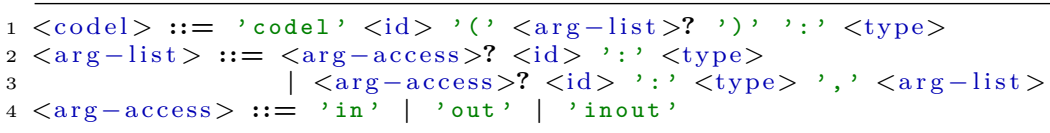

Listing 2 declares the codel for the $\mathrm{A}^{*}$ algorithm [50]: the astar function takes as argument a start pose s, a goal pose $\mathrm{g}$, a map, and the resolution of the planning graph (argument planner_resolution). It returns the path between $\mathrm{s}$ and $\mathrm{g}$.

Listing 2: Declaration of a codel for the $\mathrm{A}^{*}$ algorithm

1 codel astar (in s: PoseStamped, in $\mathrm{g}$ : PoseStamped, in map: OccupancyGrid, in planner_resolution : double): Path

\subsection{Component specification}

A component [51] is a unit of composition with contractually specified interfaces and explicit context dependencies only. A software component can be deployed independently and is subject to composition. Therefore, in order to help composition and modularity, we decompose the specification of a component into a shell and a core.

\subsubsection{Component's Shell}

The shell of a component defines its interface, i.e. its inputs and outputs. We propose four types of interfaces (see Listing 3):

- constants, which are static typed values associated to a shell. Constants are defined and initialized at the shell's specification.

- properties, which are typed component parameters, generally set at instantiation or deployment time. Properties may also have a default value. 
- data ports, similar to the push pattern proposed by [52], are used to publish data from/to a component; they are typed and oriented.

- operations, similar to the query pattern proposed by [52], are used to call functions or send requests to components; operations can be provided or required.

Listing 3: Part of the MAUVE grammar defining shells.

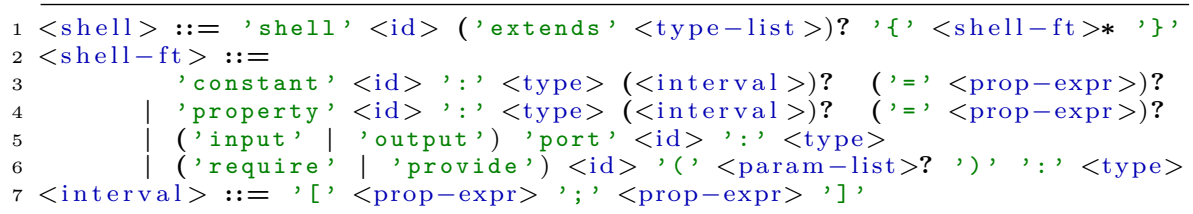

Constants and properties can only be modified from the architecture view, whereas ports and operations are used for component to component interactions. Values of properties, as well as connections of ports and operations are not initialized at the moment of specifying a component shell. Instead, they are defined when instantiating and connecting components, at the architecture specification step. Type and parameter lists are defined similarly to argument list of Listing 1. Property expressions (not described here by lack of place) allow to write simple arithmetic instructions on integers, floats and strings. Listing 4 shows the shell specification of the Navigation component. The role of this component is on one hand to compute a path from a starting position to a goal on a map, and on the other hand to manage the execution of this path by iteratively sending the path points one after the other.

Listing 4: Shell of the Navigation component

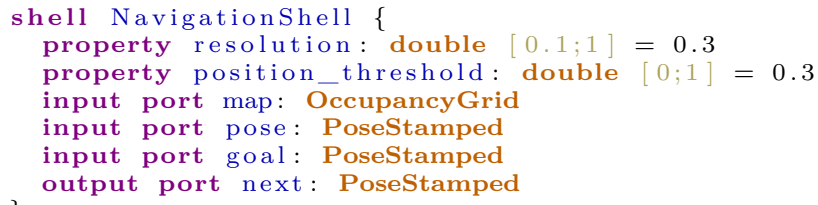

Its properties are: resolution, that specifies the resolution of the map used for path planning (in meters per cell, values between 0.1 and 1 , default to 0.3 ), and position_threshold, the threshold used to consider that the current point has been reached (in meters, values between 0 and 1 , default to 0.3 ). The component also has three input ports map (the current map known by the robot), pose (the current pose of the robot) and goal (the goal pose) and the output port next_point (on which the path points are published iteratively).

\subsubsection{Component's Core}

The core of a component defines its behaviour and has to be associated with a shell. It is defined by a state-machine. For specific cases where the state-machine only contains one state, the developer can just define the update function instead of the statemachine element. 
The core of a component is made of several elements (see Listing 5):

- variables: internal elements storing data used in the MAUVE expression language;

- provided operations: have been declared in the shell and must be defined here;

- handlers: allowing to call required operations;

- programs: five different programs can be defined to execute some computations:

- configure: this program is called to initialize the component;

- start: this program is used to start the component execution. It can only be called if the configure step has executed successfully;

- update/statemachine: this program is executed when the component is running. It can only be called if the component has been started;

- stop: this program is used to stop the execution of the component;

- cleanup: this program is called to clean the component.

Listing 5: Part of the MAUVE grammar defining cores.

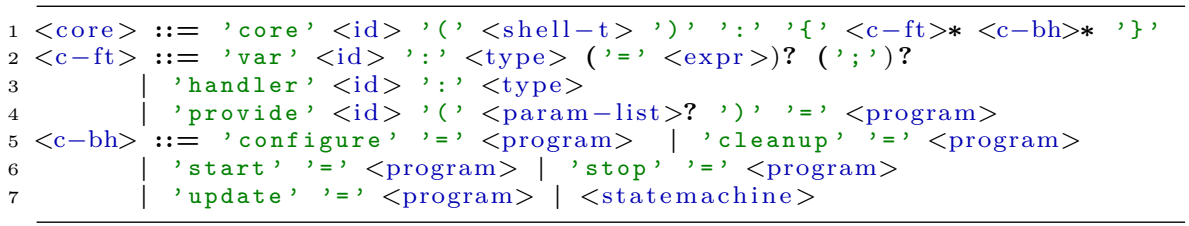

Programs are defined using a tiny expression language that allows to evaluate conditions (if/then/else), assign values to variables, get status of port (no_data, new_data, old_data), get status (failure, success, not_ready)) of handlers (remotely called required operations), call codels, read and write data on ports, call remote operations (send, collect), and use classical structures (blocks, parentheses).

Listing 6 shows the specification of the P3DX component's core and programs. The connection with the robot is initialized in configure. Data exchange with the robot is started in start. In update, data from the robot are published to output ports, and in case of a new command received in the input port command, it is sent to the robot. The robot is stopped (velocity set to 0) in stop. Finally, the connection is shutdown in cleanup.

\subsubsection{State machines}

The behaviour of each component is defined by a state machine. In case of a trivial one-state state-machine, the developer can directly define the update program as shown in Listing 6. A state-machine consists of a set of states linked by a set of transitions. MAUVE state-machine structure (see Listing 7) is very close to the UML/StateChart definition, also used in the Orocos middleware [53].

Each state $s_{i}$ contains up to four methods: entry $y_{i}$, run $_{i}$, handle $e_{i}$ and exit . The . The entry $y_{i}$ method contains code executed whenever the state-machine enters state $s_{i}$. The run $_{i}$ method contains the core of the state, executed each time the state-machine is in state $s_{i}$. The handle $e_{i}$ method is executed each time the state-machine stays in state $s_{i}$, after run $_{i}$ has been executed. The exit $t_{i}$ method is executed when leaving state $s_{i}$. Transitions are guarded and may contain a program executed when triggered. Listing 8 describes the specification of state Navigating of the Navigation component. In this state, the component executes the computed path by sending successive goals. 
Listing 6: Core of the P3DX Driver component

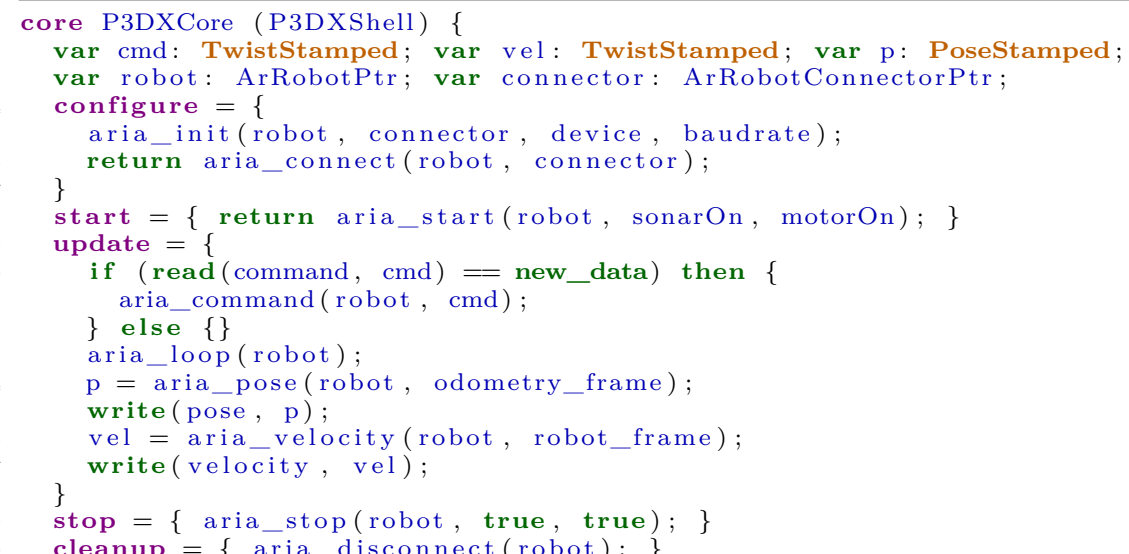

Listing 7: Part of the MAUVE grammar for state machines.

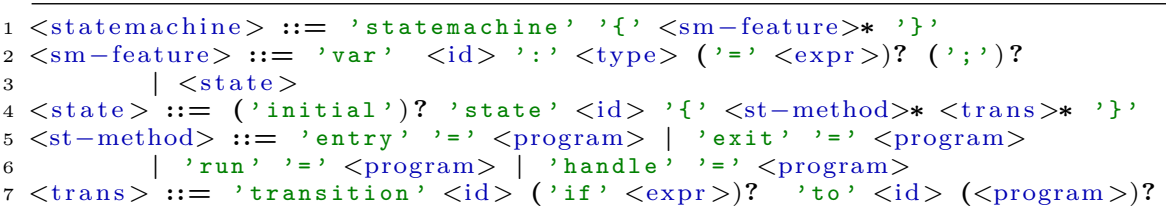

Listing 8: State Navigating of the Navigation component

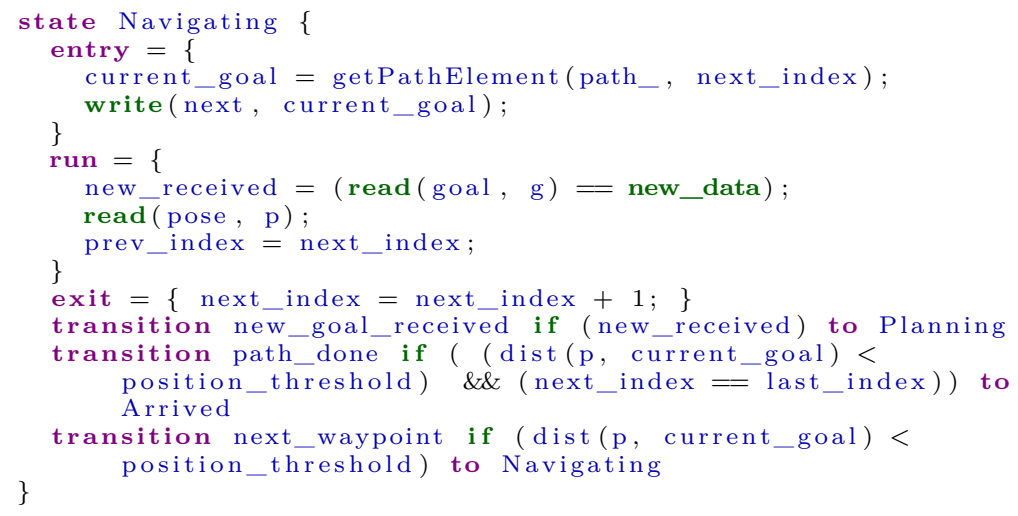

3.3 Architecture model

The architecture language (see Listing 9) allows to import an existing (partial) architecture, instantiate components, connect output ports to input ports, possibly defining the size of the buffer when the connection uses a circular buffer, connect provided operations to required operations, and set property and constant initial values. Listing 10 shows the specification the architecture drawn in Fig. 4. This architecture is made of three main components [54] designed to compute a path to follow (navigation), to avoid obstacles (guidance), and to control the robot speed (control). Other components provide sensor information (hokuyo), robot's status 


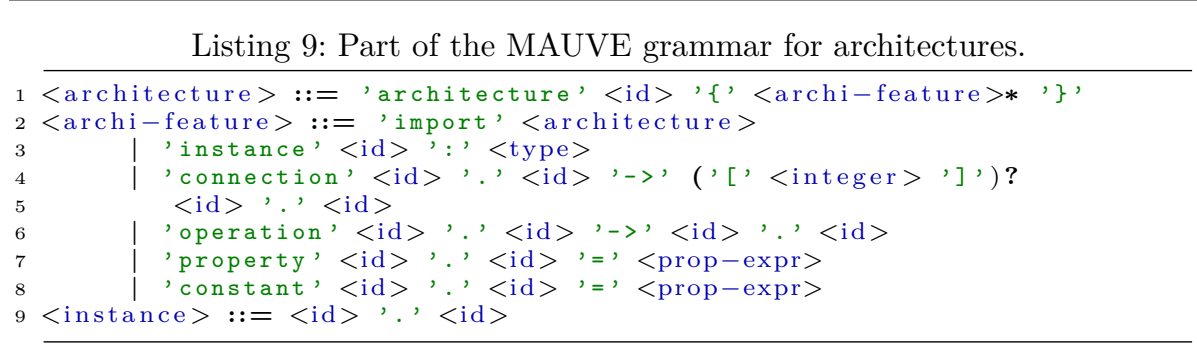

Listing 10: Exploration architecture. The architecture imports an existing navigation architecture and extends it with the exploration component and the hokuyo and P3DX driver components.

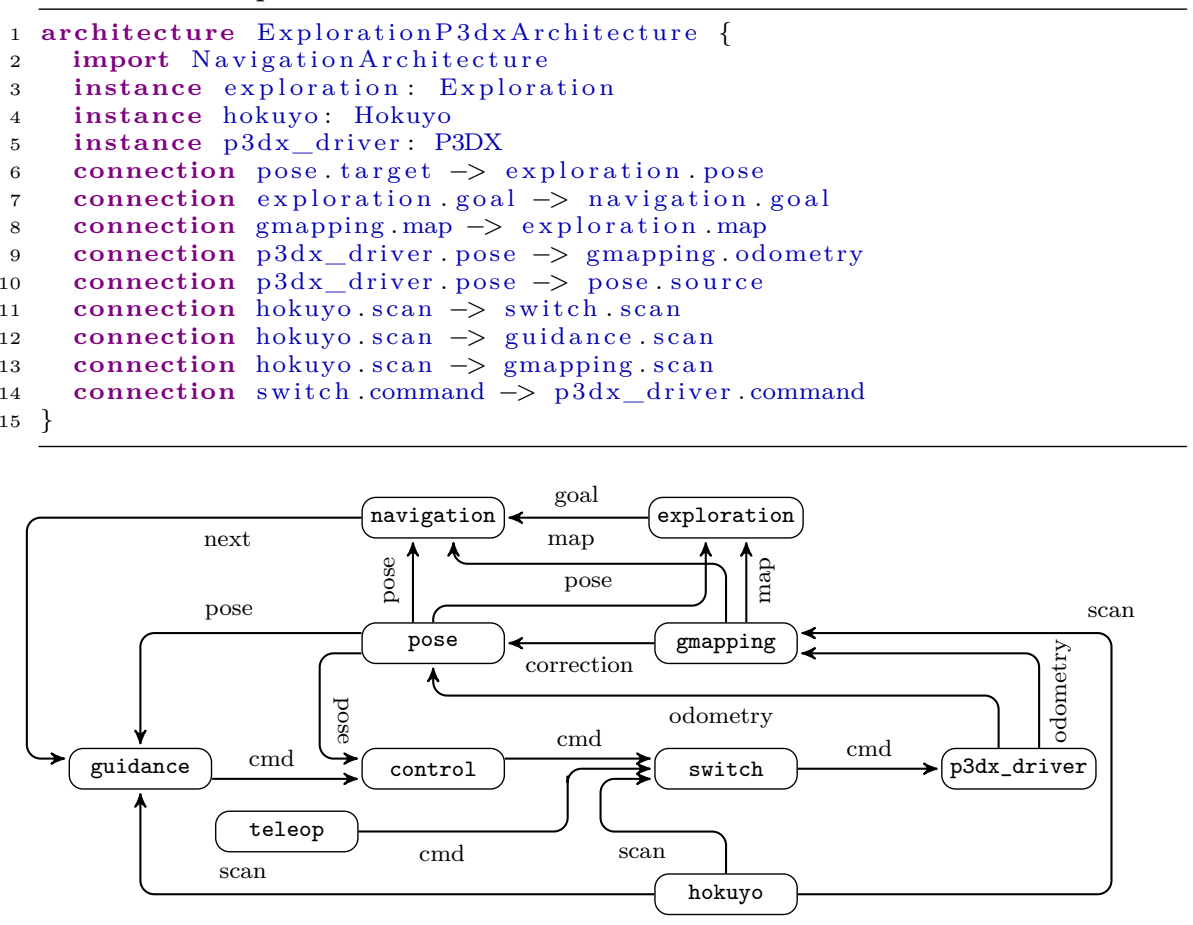

Fig. 4: The component-based exploration architecture

(p3dx_driver), or commands from a human operator (teleop). Moreover, we have connected a Simultaneous Localization And Mapping (SLAM) component (gmapping) to build a map of the environment. The pose component fuses information from SLAM and odometry to provide an accurate pose estimate. The switch component allows to select manual and automatic speed control. Finally, the exploration component selects unexplored areas on the map and send a new goal to navigate to.

\section{Software architecture deployment}

The MAUVE language has allowed to model and develop on one hand the basic components of our system, and on the other hand, architectures that assemble 
components to build applications. The next step is to deploy and execute these architectures on the final target. In this section, we present first the execution model of the components, and we then present how we can define the execution model using the MAUVE DSL deployment specification. As the task model will also need some information about the WCETs, we present some methods used to estimate WCET.

\subsection{Task model}

All the components of the software architecture are mapped onto operating system tasks. The components are designed to be temporally independent, and we then allocate each component to a specific task.

Property 1 When deploying a component, it is associated with a unique task.

Moreover the components exchange data through either asynchronous data ports and operation calls. These protocols allow a component to receive a data from another component without waiting the data to be ready.

Property 2 Data exchange between components are non-blocking.

These properties are ensured in the implementation by the Orocos middleware. Orocos first allows to execute components in threads scheduled by the OS real-time scheduler. Orocos moreover implements data sharing between components using a lock-free mechanism [55] that guarantees non blocking read/write in bounded time, and is not subject to priority inversions.

Definition 1 A component comp $p_{i}$ is executed by a task $\tau_{i}$ defined by four elements:

- the period $T_{i}$ defines the execution frequency;

- the deadline $D_{i}$ sets the moment when the task has to end its execution;

- the priority $P_{i}$ is managed by the scheduler to decide which task to execute;

- the behaviour $S M_{i}$ is defined by the internal structure of the task state-machine.

The following hypothesis is assumed true for the rest of the development.

Hypothesis 1 Deadlines are lesser or equal to the periods: $\forall i, D_{i} \leq T_{i}$

The temporal behaviour of a task depends on the activities of the tasks and on the state-machine's behaviour.

Definition 2 A state-machine $S M$ is a tuple $(\mathcal{P}, S$, E, entry, exit, handle, run, guard, exec, $\delta$ ) where:

$-\mathcal{P}$ is a set of programs; $S$ is a set of states;

$-E \subset S^{2}$ is a set of transitions where each transition $e$ is a pair $(\operatorname{src}(e), \operatorname{tgt}(e))$;

- entry $: S \rightarrow \mathcal{P}$ associates an entry program to a state;

- exit $: S \rightarrow \mathcal{P}$ associates an exit program to a state;

- run $: S \rightarrow \mathcal{P}$ associates a run program to a state;

- handle $: S \rightarrow \mathcal{P}$ associates an handle program to a state;

- guard : $E \rightarrow\{\top, \perp\}$ associates a guard expression to a transition; $\operatorname{guard}(e)$ evaluates to true $(T)$ when the transition is enabled, and false $(\perp)$ when the transition is disabled;

- exec $: E \rightarrow \mathcal{P}$ associates a program to a transition; 
$-\delta: \mathcal{P} \rightarrow \mathbb{R}$ associates an execution time to each program.

At each period of execution, two execution sequences are possible depending on the transitions that are enabled. Let's assume that current state is $s_{i}$, then:

- if no transition is triggered (2), then the component stays in state $s_{i}$, and executes $\operatorname{run}\left(s_{i}\right)$ and handle $\left(s_{i}\right)$ (Fig. 5a);

$$
\forall e \in E, \quad\left(\operatorname{src}(e)=s_{i}\right) \Rightarrow(\operatorname{guard}(e)=\perp)
$$

- if a transition $e$ is triggered (3) then the component goes from $s_{i}$ to $s_{j}=\operatorname{tgt}(e)$, and executes run $\left(s_{i}\right), \operatorname{exit}\left(s_{i}\right), \operatorname{exec}(e)$ and $\operatorname{entry}\left(s_{j}\right)$ (Fig. 5b).

$$
\left(\operatorname{src}(e)=s_{i}\right) \wedge(\operatorname{guard}(e)=\top)
$$

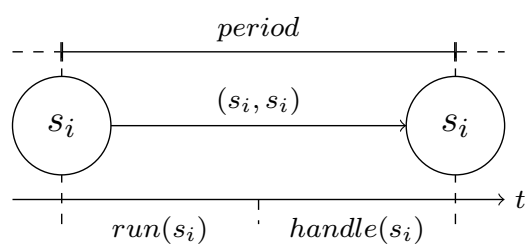

(a) when no transition is triggered

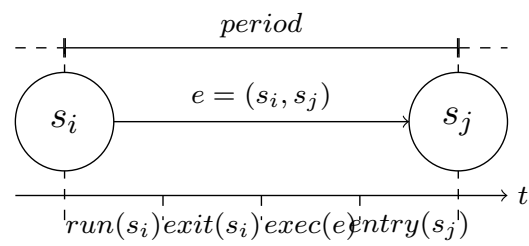

(b) when a transition occurs

Fig. 5: Execution model of state machines

4.2 Deployment specification

MAUVE allows to specify a deployment by defining the activities of each component compliant with Def. 1 . The developer can define the priority, period and deadline of each component, and allocate it to a specific CPU with the affinity (Listing 11).

Listing 11: Part of the MAUVE grammar for deployments.

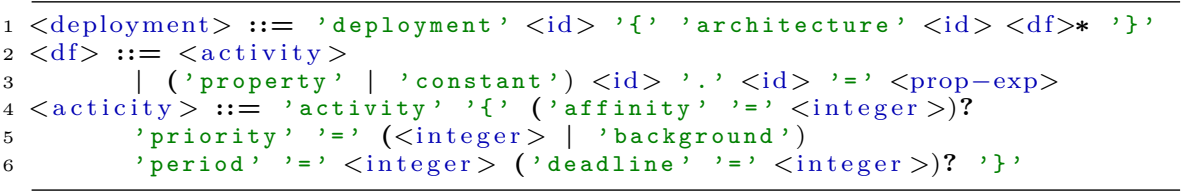

\subsection{Estimating Worst-Case Execution Times}

The task model presented in Def. 2 needs the timing function $\delta$ in order to perform the real-time analysis presented in Sect. 5. However, this execution time is generally not deterministic, and the WCET must then be estimated [56]. Static analysis [57] takes the control flow graph and a model of the hardware to compute the worst number of instructions. Static analysis methods have some limitations: (1) they need a model of each instruction, which is inappropriate when using IO functions for instance and (2) they need an accurate model of the hardware, meaning that the approach efficiency is processor-dependant. On the contrary, Measurement-Based Probabilistic Timing Analysis (MBPTA) $[58,59]$ uses measurements of execution times, and infer the probabilistic WCET (pWCET) estimation using Extreme Value Theory (EVT). Application of these methods is discussed in Sect. 7. 


\section{Real-Time analysis}

To ensure the schedulability of the task system described by the MAUVE DSL, we provide a real-time analysis process checking if the component's WCRTs are lower than their deadlines. The real-time analysis problem has been tackled many times using more and more complex approaches. The first ones were based on the Liu and Layland model [13], using fixed priority preemptive tasks, later extended to dynamic priority tasks such as EDF [14]. In order to cope with increasing application complexity, the task models are more and more refined: the Multiframe model [60, 61], the Digraph Real-Time model [62] or the Digraph Synchronous Finite StateMachine model [63] further increase the complexity and the precision of the analyses using graph based algorithms to compute WCRTs. In this section, we describe a computationally efficient and precise WCRT algorithm that is applicable to our specific task model. The proposed process (see Fig. 6) needs the WCETs of the components and of their internal codels (see Sect. 4.3). From these components we extract Periodic State-Machines (PSM) which represents the component's timed behaviour (Sect. 5.1). The WCRTs are then computed from the PSMs in three steps: the first one computes traces from the PSMs, a trace being a timeline of one possible PSM execution. These traces are used to deduce an upper bound of all the possible PSM paths, then these upper bounds allow the computation of the WCRTs (Sect. 5.2).

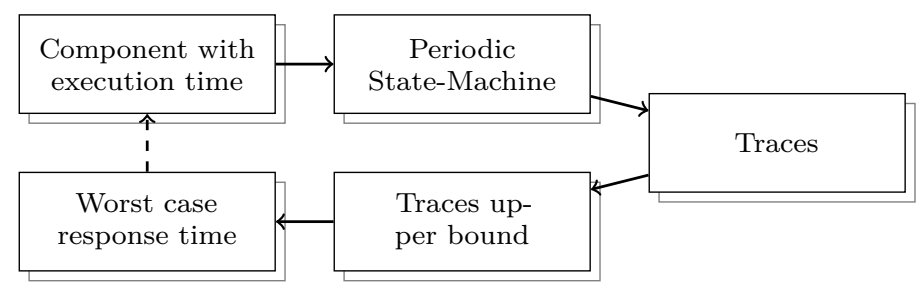

Fig. 6: Real-time analysis process

\subsection{Periodic State-Machine}

In order to analyse the components behaviour, we model them as Periodic StateMachines (PSM), with the same temporal behaviour as the task it models.

Definition 3 Each component is associated with a Periodic State-Machine PSM defined by as a set of states $S$ (similar to the states of the state-machine $S M$, see Def. 1) and a set of transitions $\Sigma$ such that:

$$
\Sigma=E \cup\{(s, s) \mid s \in S\}
$$

The set of transitions $\Sigma$ contains all the original transitions of the state-machine (E) plus all the loops over states of $S$.

Property 3 The Periodic State-Machine fires a transition at every execution period.

Property 4 A Periodic State-Machine is strongly connected: every state can be reached from any state through a sequence of transitions.

$$
\forall s_{i}, s_{j} \in S, \exists \sigma_{1} \ldots \sigma_{n} \in \Sigma \mid s_{i} \stackrel{\sigma_{1}}{\longrightarrow} \ldots \stackrel{\sigma_{n}}{\longrightarrow} s_{j}
$$


This assumption is useful, because it avoids considering that some dead states exist in the task behaviour, and it is a needed assumption for the WCRT computation (see Sect. 5.2). Moreover, this assumption is a good practice that should be enforced when developing components: it allows to be able to put the task again in its initial state at execution when a faulty behaviour occurs.

Definition 4 A timing function $\delta$ is defined over the PSM transitions, such that:

$$
\forall \sigma \in \Sigma, \sigma=\left(s_{i}, s_{j}\right), \delta(\sigma)=\left\{\begin{array}{cc}
\delta\left(\operatorname{run}\left(s_{i}\right)\right)+\delta\left(\operatorname{exit}\left(s_{i}\right)\right)+ \\
\delta\left(\operatorname{entry}\left(s_{j}\right)\right)+\delta(\operatorname{exec}(\sigma)) & \text { if } s_{i} \neq s_{j} \\
\delta\left(\operatorname{run}\left(s_{i}\right)\right)+\delta\left(\operatorname{handle}\left(s_{i}\right)\right) & \text { if } s_{i}=s_{j}
\end{array}\right.
$$

where the execution time of each program is defined in Def. 2.

\subsection{WCRT computation}

In order to compute the WCRTs (i.e., the worst time between task release and the end of its execution), we first use the PSMs structure to extract traces representing all the possible execution sequences of the state-machines.

\subsubsection{Traces}

We define a trace $\mathcal{T}$ as an ordered sequence of transitions:

$$
\mathcal{T}=\left\langle\sigma_{1}, \ldots, \sigma_{N}\right\rangle \in \Sigma^{N}
$$

We also define operators to access the $i^{\text {th }}$ transition of a trace, and the number of transitions:

$$
\begin{gathered}
\forall i \in \llbracket 1 . . N \rrbracket, \mathcal{T}[i]=\left\langle\sigma_{1}, \ldots, \sigma_{i}, \ldots, \sigma_{N}\right\rangle[i]=\sigma_{i} \\
|\mathcal{T}|=\left|\left\langle\sigma_{1}, \ldots, \sigma_{N}\right\rangle\right|=N
\end{gathered}
$$

The request bound function ( $r b f,[64])$ is the maximum processor request over a time interval. The tasks are executed periodically then the $r b f$ is a piecewise-constant and increasing function. The $r b f$ of a trace $\mathcal{T}$ at time $t$ is noted $r b f(\mathcal{T}, t)$ and is defined as:

$$
\operatorname{rbf}(\mathcal{T}, t)=\sum_{i=0}^{\left\lfloor\frac{t}{T}\right\rfloor} \delta(\mathcal{T}[i+1])
$$

with $\delta(\sigma)$ the time taken by transition $\sigma$ (see Def. 4). We then define the relation $\leq$ on traces by comparing the $r b f$ of the traces:

$$
\mathcal{T} \leq \mathcal{T}^{\prime} \Leftrightarrow \forall t, r b f(\mathcal{T}, t) \leq \operatorname{rbf}\left(\mathcal{T}^{\prime}, t\right)
$$

The next operator represents the set of traces that follows a given trace:

$$
\operatorname{next}(\mathcal{T})=\{\langle\mathcal{T}, \sigma\rangle \mid \sigma \in \Sigma \wedge \operatorname{tgt}(\mathcal{T}[|\mathcal{T}|])=\operatorname{src}(\sigma)\}
$$




\subsubsection{Traces upper bound}

WCRT of tasks relies on the computation of an upper bound of all the feasible traces of a task with respect to their $r b f$. According to relation (11), we only need to reason on the maximal feasible traces: two traces arriving at the same state will be extended the same way (12). Therefore we only need to keep the greatest trace among the set of traces that terminate in the same state. We then compute the set of traces that are maximal using the following recursive equations:

$$
\begin{gathered}
\mathcal{V}^{1}=\left\{\langle\sigma\rangle, \sigma \in T \mid \forall \sigma^{\prime} \in T, \sigma \neq \sigma^{\prime} \wedge\left(\operatorname{tgt}(\sigma)=\operatorname{tgt}\left(\sigma^{\prime}\right) \Longrightarrow\langle\sigma\rangle \geq\left\langle\sigma^{\prime}\right\rangle\right)\right\} \\
\mathcal{V}^{n+1}=\left\{\langle\mathcal{T}, \sigma\rangle, \mathcal{T} \in \mathcal{V}^{n}, \sigma \in T \mid \forall \mathcal{T}^{\prime} \in \mathcal{V}^{n}, \forall \sigma^{\prime} \in T, \quad \mathcal{T}^{\prime} \neq \mathcal{T} \wedge\langle\mathcal{T}, \sigma\rangle \in \operatorname{next}(\mathcal{T})\right. \\
\left.\wedge\left\langle\mathcal{T}^{\prime}, \sigma^{\prime}\right\rangle \in \operatorname{next}\left(\mathcal{T}^{\prime}\right) \wedge\left(\operatorname{tgt}(\sigma)=\operatorname{tgt}\left(\sigma^{\prime}\right) \Longrightarrow\langle\mathcal{T}, \sigma\rangle \geq\left\langle\mathcal{T}^{\prime}, \sigma^{\prime}\right\rangle\right)\right\}
\end{gathered}
$$

From these trace set, we can extract $\mathcal{T}^{+}$, an upper bound of all the feasible traces of the task according to (11) using the following recursive equations:

$$
\begin{aligned}
\mathcal{T}_{1}^{+} & =\langle\sigma\rangle \mid \sigma=\underset{\sigma_{i} \in \Sigma}{\operatorname{argmax}} \delta\left(\sigma_{i}\right) \\
\mathcal{T}_{n+1}^{+} & =\left\langle\mathcal{T}_{n}^{+}, \mathcal{T}[n+1]\right\rangle \mid \mathcal{T}=\underset{\mathcal{T}^{\prime} \in \mathcal{V}^{n+1}}{\operatorname{argmax}} \operatorname{rbf}\left(\mathcal{T}^{\prime},(n+1) \times T\right)
\end{aligned}
$$

The construction of trace $\mathcal{T}^{+}$does not ensure that the trace is feasible, i.e. that two consecutive transitions of the trace are effectively consecutive transitions of the PSM. The timing function $\delta$ has therefore no meaning on $\mathcal{T}^{+}$and the computation of the $r b f$ on $\mathcal{T}^{+}$must use a new function $\delta^{+}$recursively defined while building $\mathcal{T}^{+}$by:

$$
\begin{aligned}
\delta^{+}\left(\mathcal{T}^{+}[1]\right) & =\delta(\sigma) \\
\delta^{+}\left(\mathcal{T}^{+}[n+1]\right) & =\operatorname{rbf}(\mathcal{T}, n+1)-\sum_{j=1}^{n} \delta^{+}\left(\mathcal{T}^{+}[j]\right)
\end{aligned}
$$

with $\sigma$ and $\mathcal{T}$ respectively defined in (15) and (16).

\subsubsection{WCRT}

In order to compute the WCRT, we adapted the usual recursive procedure proposed by [13]. Instead of using the classical task's execution time, we use the upper bound trace's values: at each iteration of the recursive procedure, we use the next iteration of the upper bound trace.

$$
\begin{aligned}
\mathcal{R}_{i}^{0} & =\operatorname{rbf}\left(\mathcal{T}_{i}^{+}, 0\right) \\
\mathcal{R}_{i}^{n+1} & =\sum_{j \in h p(i)} \operatorname{rbf}\left(\mathcal{T}_{j}^{+}, \mathcal{R}_{i}^{n}\right)+\mathcal{R}_{i}^{0}
\end{aligned}
$$

In equation (20), the sum is made over all traces $\mathcal{T}_{j}^{+}$that have a greater priority than the $i$-th component (i.e., $j \in h p(i)$ ). Two conditions stop this recursive loop: (1) whenever two consecutive iterations have the same $\mathcal{R}_{i}$ value, (2) whenever $\mathcal{R}_{i}^{n+1}$ reaches the task's deadline $D_{i}$. In the latter the component is not schedulable. Finally, if all the WCRT are lesser than the deadlines (i.e., $\forall i, \mathcal{R}_{i} \leq D_{i}$ ), the complete software architecture is schedulable. 


\section{Code generation}

In order to eventually execute the architecture on the target platform, we have set up a code generation process (see Fig. 7). It takes as input the MAUVE models of

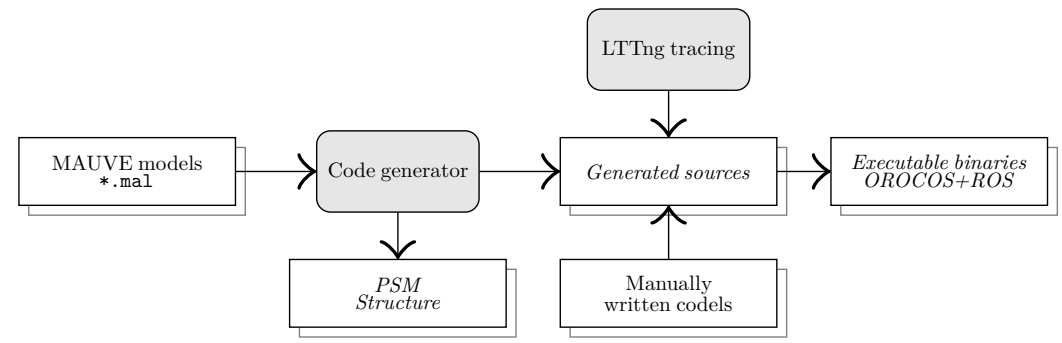

Fig. 7: Code generation process

components and architectures specified using the MAUVE DSL (see Sect. 3). The code generator then translates this model into source files and PSM models of the components (see Sect. 5.1) in order to apply the analysis process. The source files are completed with the definition of codels, that can either by directly implemented in the generated files, or linked with a third-party library. The resulting software architecture uses the ROS ecosystem for compiling the software, and launch files to help the deployment. The software architecture is only made of Orocos components: Orocos manages the real-time execution and data exchange between components. The generated sources also embed a tracing library, based on LTTng [65], that allows to produce tracepoints at runtime, including clock values and relevant data about the execution of components (start and end of states and programs, codel calls).

\section{Results and discussion}

The methodology presented in this paper has been fully applied to the application presented in Sect. 2. The deployment specification of the architecture of Fig. 4 is presented in Tab. 2. The period of the p3dx_driver is based on the period of the communication with the low-level processor embedded on the robot. Most of the periods are then set to $100 \mathrm{~ms}$. The navigation and gmapping components are executed with a period of $1 \mathrm{~s}$, as they may be quite time consuming. Moreover, the gmapping component is isolated on the second CPU core. The exploration component is set non real-time: it is executed sporadically when a new exploration point has to be defined. ${ }^{1}$

\section{1 pWCET estimations}

We have first applied the pWCET estimation method based on measurements to obtain WCETs of component functions. The MBPTA approach (see Sect. 4.3) uses

\footnotetext{
1 a non real-time component is not scheduled by the real-time scheduler, and consequently does not disturb the behaviour of real-time components.
} 
Table 2: Deployment specification

\begin{tabular}{c|r|r|c|c|} 
component & period $(\mathrm{ms})$ & deadline $(\mathrm{ms})$ & priority & CPU \\
\hline \hline p3dx_driver & 100 & 100 & 10 & 1 \\
\hline hokuyo & 100 & 100 & 9 & 1 \\
\hline switch & 100 & 100 & 8 & 1 \\
\hline pose & 100 & 100 & 7 & 1 \\
\hline guidance & 100 & 100 & 6 & 1 \\
\hline control & 100 & 100 & 5 & 1 \\
\hline teleop & 100 & 100 & 4 & 1 \\
\hline navigation & 1000 & 1000 & 2 & 1 \\
\hline gmapping & 1000 & 1000 & 3 & 2 \\
\hline exploration & - & - & background & - \\
\hline
\end{tabular}

measures of execution times of the codels, independently of the deployment that is executed. It is then possible to perform independent measurements from several deployments and executions:

- an automatic control deployment, in which we deployed the p3dx_driver, control, hokuyo, switch and guidance components; we manually sent some guidance goals to the guidance component in a good variability of input conditions such as a densely packed storage area with complex shapes, tight straight corridors and a wide open room with and without moving objects;

- a mapping deployment, in which we deployed the necessary components to manually control the robot from user command, while building a map;

- a navigation deployment, in which we only deployed the navigation component; the component has been fed with a static global map; we randomly sent several goals to the navigation component without moving the robot.

The exploration component has not been used: it is not executed in real-time, and then is not considered in the real-time analysis. The p3dx_driver state machine has only one state (Listing 6). This state executes a set of codels, for which we have to estimate the WCET. Figure 8a shows some measurements of the execution time of the aria_loop codel, on a small part of the gathered measures.

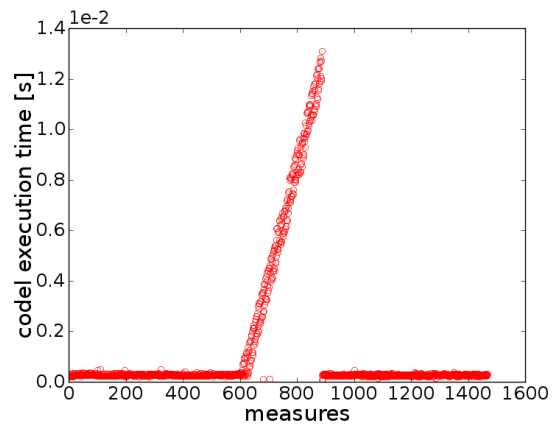

(a) Measurements of codel execution

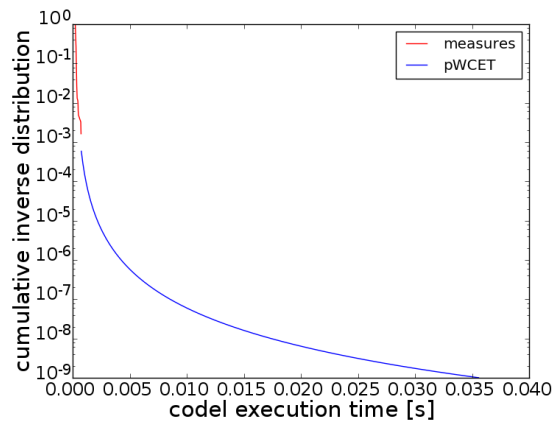

(b) pWCET estimation

Fig. 8: MBPTA applied to the aria_loop codel 
This codel is responsible of making the actual communication with the robot controller through a serial connection. Therefore, the communication time with the hardware is taken into account into the measurements. The pWCET estimation of the aria_loop codel is shown in Fig. 8b. From this pWCET estimation, the WCET of the p3dx_driver component is estimated according to several possible significance thresholds. Figure 9 shows the PSM for a significance level of $10^{-7}$.

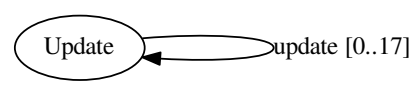

Fig. 9: PSM of the p3dx_driver component for a significance level of $10^{-7}$

\subsection{Schedulability results}

From the MAUVE models and the estimated WCETs, we have built the PSM models for all the components and all the desired significance levels.

\subsubsection{Evaluating the impact of $p$ WCET threshold}

Table 3 shows the resulting WCRT for the several components according to the selected significance level. At $10^{-5}$, the navigation component cannot be proved to be schedulable. At $10^{-9}$, only the p3dx_driver component is schedulable. As the hokuyo WCRT overshoots its deadline, it is not possible to compute a WCRT for the less priority components. The gmapping component runs on a separate CPU core. The component is schedulable down to a significance of $10^{-7}$.

Table 3: WCRT results for all components running on the CPU. Values are in milliseconds. Values in bold are exceeding the component deadline.

\begin{tabular}{|c|c|c|c|c|c|c|c|}
\hline $\begin{array}{c}\text { Core } 1 \\
\text { component }\end{array}$ & $1 e-3$ & $1 \mathrm{e}-4$ & $1 e-5$ & $1 \mathrm{e}-6$ & $1 \mathrm{e}-7$ & $1 e-8$ & $1 \mathrm{e}-9$ \\
\hline p3dx_driver & 8 & 10 & 11 & 13 & 17 & 29 & 55 \\
\hline hokuyo & 48 & 53 & 55 & 58 & 63 & 75 & 156 \\
\hline switch & 51 & 56 & 58 & 63 & 69 & 84 & - \\
\hline pose & 52 & 57 & 59 & 64 & 70 & 85 & - \\
\hline guidance & 55 & 60 & 62 & 67 & 73 & 88 & - \\
\hline control & 58 & 63 & 65 & 70 & 76 & 91 & - \\
\hline teleop & 65 & 70 & 72 & 77 & 83 & 98 & - \\
\hline navigation & 882 & 997 & 1017 & 1067 & 1127 & 1277 & - \\
\hline $\begin{array}{c}\text { Core } 2 \\
\text { component }\end{array}$ & $1 \mathrm{e}-3$ & $1 \mathrm{e}-4$ & $1 \mathrm{e}-5$ & $1 e-6$ & $1 e-7$ & $1 e-8$ & $1 \mathrm{e}-9$ \\
\hline gmapping & 789 & 789 & 791 & 798 & 839 & 1069 & 2333 \\
\hline
\end{tabular}

\subsubsection{Evaluating the impact of state-machines}

The method we have presented in this paper takes explicitly state-machines into account when computing the WCRT of components using an upper bound of traces 
$\mathcal{T}^{+}$(let's note this WCRT $\mathcal{R}^{+}$). We have compared with the computation of the WCRT using the classical method, i.e. by considering that each task has only one WCET. It corresponds to defining for each component a PSM with only one state (like in Fig. 9), whose WCET would be the maximum of the WCET values of the original PSM transitions. Let's note this WCRT $\mathcal{R}^{*}$. The $\mathcal{R}^{*}$ for the components running on CPU first core are shown in Tab. 4.

Table 4: $\mathcal{R}^{*}$ for components running on core 1 . Values are in milliseconds. Values in bold are exceeding the component deadline.

\begin{tabular}{c|r|r|r|r|r|r|r|} 
component & $1 \mathrm{e}-3$ & $1 \mathrm{e}-4$ & $1 \mathrm{e}-5$ & $1 \mathrm{e}-6$ & $1 \mathrm{e}-7$ & $1 \mathrm{e}-8$ & $1 \mathrm{e}-9$ \\
\hline \hline p3dx_driver & 8 & 10 & 11 & 13 & 17 & 29 & 55 \\
\hline hokuyo & 48 & 53 & 55 & 58 & 63 & 75 & $\mathbf{1 5 6}$ \\
\hline switch & 51 & 57 & 59 & 64 & 70 & 85 & - \\
\hline pose & 52 & 58 & 60 & 65 & 71 & 86 & - \\
\hline guidance & 55 & 61 & 63 & 68 & 74 & 89 & - \\
\hline control & 58 & 64 & 66 & 71 & 77 & 92 & - \\
\hline teleop & 65 & 71 & 73 & 78 & 84 & 99 & - \\
\hline navigation & 884 & $\mathbf{1 0 8 0}$ & $\mathbf{1 1 7 5}$ & $\mathbf{1 3 9 1}$ & $\mathbf{1 8 9 5}$ & $\mathbf{2 9 9 0 0}$ & - \\
\hline
\end{tabular}

Regarding the schedulability of components, the only noticeable situation is for the navigation component at a level of $10^{-4}$ : it can be proved schedulable using $\mathcal{R}^{+}$ but not with $\mathcal{R}^{*}$. The gain obtained with our method can be computed as:

$$
\Gamma=\frac{\mathcal{R}^{*}-\mathcal{R}^{+}}{\mathcal{R}^{*}}
$$

The value of $\Gamma$ for the navigation component (i.e. for the whole architecture as the navigation is the less priority component) is 0.077 for a significance level of $10^{-4}$ (meaning that our method is around $7 \%$ less pessimistic), and up to 0.957 for a level of $10^{-8}$ (meaning that our method is about $95 \%$ less pessimistic). The gain is clearly greater when considering components with state machines that have quite different WCET values, like for the navigation component, where the WCET of transitions is of $297 \mathrm{~ms}$ when executing the astar codel, while other transitions have a WCET lower than $3 \mathrm{~ms}$.

\subsection{Discussion}

The results of Tab. 3 lead to the following conclusions:

- the whole architecture is schedulable if a significance level of $10^{-4}$ is acceptable;

- if we need a lower level, down to $10^{-7}$, all the architecture is proved to be schedulable, but the navigation component.

- at a significance level of $10^{-8}$, all the architecture is proved to be schedulable, but the navigation and gmapping components.

In the other cases, nothing can be deduced regarding the schedulability: we compute an upper bound of the WCRT, and then we cannot conclude that the components are actually schedulable or not.

Besides the direct conclusion regarding schedulability, these results can help designing the architecture. They indeed emphasize some real-time behaviours that 
may happen during an actual run of the system. Let us consider that we would like to provide a significance level of $10^{-8}$. Some possible design choices may be to:

- do nothing: gmapping and navigation are the less priority components on their respective core, and in the case where they would pass their deadline, they will have no impact on the other components;

- change the affinity of the navigation component: by moving it to a core where it will be the only real-time component, it may be proved to be schedulable, which may be mandatory if its functions are critical for the safety of the system;

- let some components run in background mode: they will not be real-time any more, meaning that they may pass their deadline, but also that they may run faster than their period when the CPU is under loaded; it may lead to more efficient applications but can be done only for non safety critical components.

In the context of safety critical applications, it is mandatory to perform such real-time analyses to ensure that the system cannot produce unsafe behaviours. More importantly our work allows the designer to apprehend the real-time behaviour of its application and take it into account in the design phase. In the case of the P3-DX robot, violating real-time constraints may for instance lead to decreasing the localization quality (in case the map computed by the gmapping component is distorded), or to possible collisions in case the guidance or switch components are delayed.

\section{Conclusion}

Safety critical applications demand for sound development processes and for analyses that help proving that the system cannot produce unsafe behaviours. Such analyses must encompass both the functionnal properties, i.e., verifying that the algorithms provide the good results, and the non-functionnal properties, among which verifying that the algorithms execute on time. In this paper, we have focused on verifying such real-time constraints as they are necessary to guarantee a correct and safe behavior of the whole system.

In this paper, we have presented a complete toolchain for the design and analysis of component-based real-time software architectures. This toolchain settles on the MAUVE DSL to model components, architectures and deployments. The toolchain comes with code generation towards Orocos real-time tasks. The generated code moreover embeds tracepoints to provide timed traces of execution runs. The real-time analysis takes benefit of the behaviour of components to compute a WCRT less pessimistic than classical approaches that consider components as a unique function.

We have illustrated the whole process on the design of a software architecture for a ground robot performing an exploration mission. We have first shown the architecture design aspects, with code snapshots of the MAUVE models of components and architectures. We have then discussed and illustrated how timed traces can be used to estimate the WCET of the elementary functions called by each component. Finally, we have run the complete real-time analysis process, and discussed how the results can led either to prove the real-time safety of critical parts of the architecture, and help the designer make some conception choices about its deployment. 
Future works are twofold. Regarding the real-time analysis, it would be interesting to avoid selecting a significance level before computing the WCRT, and we are working on how to directly propagate the pWCET continuous law inside the WCRT computation in order to compute a pWCRT. It would lead to even less pessimistic results as some probabilistic dependency between components and/or states could be taken into account.

Secondly, the WCRT computation uses an execution model that is over pessimistic because we suppose that every task clock are not synchronized; removing this assumption would lead to less complexity in the computation of traces upper bounds and WCRT; This behaviour is due to some limitations of the Orocos middleware regarding real-time synchronisation, and we are considering to make the middleware evolve to fix this behaviour [66].

\section{References}

1. Gustavo Freitas, Bradley Hamner, Marcel Bergerman, and Sanjiv Singh. A practical obstacle detection system for autonomous orchard vehicles. In Int. Conf. on Intelligent Robots and Systems (IROS), Vilamoura-Algarve, Portugal, 2012.

2. Sami Haddadin, Simon Haddadin, Augusto Khoury, Tim Rokahr, Sven Parusel, Rainer Burgkart, Antonio Bicchi, and Alin Albu-Schaffer. A truly safely moving robot has to know what injury it may cause. In Int. Conf. on Intelligent Robots and Systems (IROS), Vilamoura-Algarve, Portugal, 2012.

3. Thomas Lens and Oskar Von Stryk. Investigation of safety in human-robot-interaction for a series elastic, tendon-driven robot arm. In Int. Conf. on Intelligent Robots and Systems (IROS), Vilamoura-Algarve, Portugal, 2012.

4. Paul Rybski, Peter Anderson-Sprecher, Daniel Huber, Chris Niessl, and Reid Simmons. Sensor fusion for human safety in industrial workcells. In Int. Conf. on Intelligent Robots and Systems (IROS), Vilamoura-Algarve, Portugal, 2012.

5. Yusuke Tamura, Phuoc Dai Le, Kentarou Hitomi, Naiwala Chandrasiri, Takashi Bando, Atsushi Yamashita, and Hajime Asama. Development of pedestrian behavior model taking account of intention. In Int. Conf. on Intelligent Robots and Systems (IROS), Vilamoura-Algarve, Portugal, 2012.

6. Sebastian Elbaum and Carrick Detweiler. Reducing failure rates of robotic systems though inferred invariants monitoring. In Int. Conf. on Intelligent Robots and Systems (IROS), Tokyo, Japan, 2013.

7. Akira Nakamura, Kazuyuki Nagata, Kensuke Harada, Natsuki Yamanobe, Tokuo Tsuji, Torea Foissotte, and Yoshihiro Kawai. Error recovery using task stratification and error classification for manipulation robots in various fields. In Int. Conf. on Intelligent Robots and Systems (IROS), Tokyo, Japan, 2013.

8. Jonathan Decastro and Hadas Kress-Gazit. Guaranteeing reactive high-level behaviors for robots with complex dynamics. In Int. Conf. on Intelligent Robots and Systems (IROS), Tokyo, Japan, 2013.

9. Shashank Pathak, Luca Pulina, Giorgio Metta, and Armando Tacchella. Ensuring safety of policies learned by reinforcement: Reaching objects in the presence of obstacles with the iCub. In Int. Conf. on Intelligent Robots and Systems (IROS), Tokyo, Japan, 2013.

10. Anton Cervin, Bo Lincoln, Johan Eker, Karl-Erik Årzén, and Giorgio Buttazzo. The Jitter Margin and Its Application in the Design of Real-Time Control Systems. In Int. Conf. on Real-Time and Embedded Computing Systems and Applications (RTCSA), Göteborg, Sweden, 2004.

11. Dan Henriksson, Anton Cervin, Johan Åkesson, and Karl-Erik Årzén. On Dynamic RealTime Scheduling of Model Predictive Controllers. In Int. Conf. on Decision and Control $(C D C)$, Las Vegas, NV, USA, 2002.

12. Mark H Klein, Thomas Ralya, Bill Pollak, Ray Obenza, and Michael González Harbour. A practitioner's handbook for real-time analysis. Kluwer Academic Publishers, 1993.

13. L Liu, C James, and W Layland. Scheduling Algorithms for Multiprogramming in a HardReal-Time Environment Scheduling Algorithms for Multiprogramming. Journal of the Association for Computing Machinery, 20(1), jan 1973. 
14. Marco Spuri. Analysis of Deadline Scheduled Real-Time Systems. Technical report, 1996.

15. Lui Sha, Ragunathan Rajkumar, and John P Lehoczky. Priority inheritance protocols: An approach to real-time synchronization. IEEE Trans. on Computers, 39(9), 1990.

16. Min-Ih Chen and Kwei-Jay Lin. Dynamic priority ceilings: A concurrency control protocol for real-time systems. Real-Time Systems, 2(4), 1990.

17. Theodore P Baker. Stack-based scheduling of realtime processes. Real-Time Systems, 3(1), 1991.

18. Houssine Chetto, M Silly, and T Bouchentouf. Dynamic scheduling of real-time tasks under precedence constraints. Real-Time Systems, 2(3), 1990.

19. Erann Gat. On three-layer architectures. In Artificial intelligence and mobile robots. 1998.

20. James S Albus. 4D/RCS: a reference model architecture for intelligent unmanned ground vehicles. AeroSense 2002, 2002.

21. C McGann, F Py, K Rajan, H Thomas, R Henthorn, and R McEwen. A deliberative architecture for AUV control. In Int. Conf. on Robotics and Automation (ICRA), Pasadena, California, USA, 2008.

22. Nicola Muscettola, Gregory Dorais, Chuck Fry, Richard Levinson, and Christian Plaunt. IDEA: Planning at the Core of Autonomous Reactive Agents. In Int. NASA Workshop on Planning and Scheduling for Space (IWPSS), Houston, TX, USA, 2002.

23. Richard Volpe, Issa Nesnas, Tara Estlin, D Mutz, Richard Petras, and Hari Das. The CLARAty architecture for robotic autonomy. In IEEE Aerospace Conf., Big Sky, MT, USA, 2001.

24. Davide Brugali and Patrizia Scandurra. Component-Based Robotic Engineering (Part I). IEEE Robotics and Automation Magazine, 16(4), 2009.

25. Davide Brugali and Azamat Shakhimardanov. Component-Based Robotic Engineering (Part II). IEEE Robotics and Automation Magazine, 17(1), 2010.

26. Min Yang Jung, Marcin Balicki, Russell H Taylor, and Peter Kazanzides. Lessons Learned from the Development of Component-Based Medical Robot Systems. Journal of Software Engineering for Robotics (JOSER), 5(2), 2013.

27. Noriaki Ando, Shinji Kurihara, Geoffrey Biggs, Takeshi Sakamoto, Hiroyuki Nakamoto, and Tetsuo Kotoku. Software Deployment Infrastructure for Component Based RT-Systems. Journal of Robotics and Mechatronics, 23(3), 2011.

28. Luis Manso, Pilar Bachiller, Pablo Bustos, Pedro Nunez, Ramon Cintas, and Luis Calderita. RoboComp: A Tool-Based Robotics Framework. In International Conference on Simulation, Modelling and Programming for Autonomous Robots (SIMPAR), Darmstadt, Germany, 2010.

29. Jesús Martínez, Adrián Romero-Garcés, Luis Manso, and Pablo Bustos. Improving a robotics framework with real-time and high-performance features. In Int. Conf. on Simulation, Modeling, and Programming for Autonomous Robots (SIMPAR), Darmstadt, Germany, 2010.

30. Peter Soetens and Herman Bruyninckx. Realtime hybrid task-based control for robots and machine tools. In Int. Conf. on Robotics and Automation (ICRA), Barcelona, Spain, 2005.

31. Morgan Quigley, Ken Conley, Brian Gerkey, Josh Faust, Tully Foote, Jeremy Leibs, Eric Berger, Rob Wheeler, and Andrew Mg. ROS: an open-source Robot Operating System. In ICRA Workshop on Open Source Software, Kobe, Japan, 2009.

32. Ananda Basu, Matthieu Gallien, Charles Lesire, Thanh-hung Nguyen, Saddek Bensalem, Félix Ingrand, and Joseph Sifakis. Incremental Component-based Construction and Verification of a Robotic System. In Eureopean Conf. on Artificial Intelligence (ECAI), Patras, Greece, 2008.

33. Nicolas Navet and Loïc Fejoz. CPAL: High-level Abstractions for Safe Embedded Systems. In Proceedings of the International Workshop on Domain-Specific Modeling, DSM 2016, pages 35-41, New York, NY, USA, 2016. ACM.

34. http://www.rock-robotics.org/stable/. Rock, the robot construction kit.

35. Herman Bruyninckx, Markus Klotzbücher, Nico Hochgeschwender, Gerhard Kraetzschmar, Luca Gherardi, and Davide Brugali. The BRICS Component Model: A Model-Based Development Para-digm For Complex Robotics Software Systems. In ACM Symposium on Applied Computing (SAC), Coimbra, Portugal, 2013.

36. Anthony Mallet, Cédric Pasteur, and Matthieu Herrb. GenoM3: Building middlewareindependent robotic components. In Int. Conf. on Robotics and Automation (ICRA), Anchorage, AK, USA, may 2010. 
37. Mohammed Foughali, Bernard Berthomieu, Silvano Dal Zilio, Félix Ingrand, and Anthony Mallet. Model Checking Real-Time Properties on the Functional Layer of Autonomous Robots. In International Conference on Formal Engineering Methods (ICFEM), Tokyo, Japan, 2016.

38. Christian Schlegel, Andreas Steck, Davide Brugali, and Alois Knoll. Design Abstraction and Processes in Robotics: From Code-Driven to Model-Driven Engineering. In Int. Conf. on Simulation, Modeling, and Programming for Autonomous Robots (SIMPAR), Darmstadt, Germany, 2010.

39. Andreas Steck and Christian Schlegel. Towards quality of service and resource aware robotic systems through model-driven software development. In Int. Workshop on Domain-Specific Languages and models for Robotic systems (DSLRob), Taipei, Taiwan, 2010.

40. F. Singhoff, J. Legrand, L. Nana, and L. Marcé. Cheddar : a Flexible Real Time Scheduling Framework. ACM SIGAda Ada Letters, 24(4):1-8, 2004.

41. Nicolas Gobillot, Charles Lesire, and David Doose. A Modeling Framework for Software Architecture Specification and Validation. In Int. Conf. on Simulation, Modeling, and Programming for Autonomous Robots (SIMPAR), Bergamo, Italy, 2014.

42. Nicolas Gobillot, David Doose, Charles Lesire, and Luca Santinelli. Periodic state-machine aware real-time analysis. In Emerging Technologies and Factory Automation (ETFA), Luxembourg, Luxembourg, 2015.

43. Nicolas Gobillot, Fabrice Guet, David Doose, Christophe Grand, Charles Lesire, and Luca Santinelli. Measurement-based real-time analysis of robotic software architectures. In Int. Conf. on Intelligent Robots and Systems (IROS), Daejeon, South Korea, 2016.

44. Yoko Watanabe, Augustin Manecy, Alexandre Amiez, Charles Lesire, and Christophe Grand. Non-cooperative ground vehicle tracking and interception by multi-RPA collaboration. In International Council of the Aeronautical Sciences (ICAS), Daejeon, South Korea, 2016.

45. Peter Feiler and David Gluch. Model-Based Engineering with AADL: An Introduction to the SAE Architecture Analysis \&5 Design Language. Addison-Wesley Professional, 2012.

46. Dániel Varró. A Formal Semantics of UML Statecharts by Model Transition Systems. In Int. Conf. on Graph Transformation (ICGT), Barcelona, Spain, 2002.

47. Bran Selic and Sébastien Gérard. Modeling and Analysis of Real-Time and Embedded Systems with UML and MARTE: Developing Cyber-Physical Systems. Elsevier, 2013.

48. Geoffrey Biggs, Kiyoshi Fujiwara, and Keiju Anada. Modelling and Analysis of a Redundant Mobile Robot Architecture Using AADL. In Int. Conf. on Simulation, Modeling, and Programming for Autonomous Robots (SIMPAR), Bergamo, Italy, 2014.

49. Gianluca Bardaro, Andrea Semprebon, and Matteo Matteucci. AADL for robotics: a general approach for system architecture modeling and code generation. Journal of Software Engineering for Robotics (JOSER), 8(1):32-44, 2017.

50. Peter Hart, Nils Nilsson, and Bertram Raphael. A Formal Basis for the Heuristic Determination of Minimum Cost Paths. IEEE Trans. on Systems, Science, and Cybernetics, 4(2), 1968.

51. Clemens Szyperski, Dominik Gruntz, and Murer Stephan. Component Software: Beyond Object-Oriented Programming. Reading, MA : Addison-Wesley, 202.

52. Christian Schlegel. Communication Patterns as Key Towards Component-Based Robotics. Journal of Advanced Robotic Systems, 3(1), 2006.

53. Markus Klotzbücher and Herman Bruyninckx. Coordinating Robotic Tasks and Systems with rFSM Statecharts. Journal of Software Engineering for Robotics (JOSER), 1(1), 2012 .

54. Nicolas Gobillot, Charles Lesire, and David Doose. A Component-Based NavigationGuidance-Control Design Pattern for Mobile Robots. In ICRA Workshop on Software Development and Integration for Robotics (SDIR), Karlsruhe, Germany, 2013.

55. Maurice Herlihy. Wait-free Synchronization. ACM Transactions on Programming Languages and Systems, 13(1):124-149, 1991.

56. Reinhard Wilhelm, Tulika Mitra, Frank Mueller, Isabelle Puaut, Peter Puschner, Jan Staschulat, Per Stenström, Jakob Engblom, Andreas Ermedahl, Niklas Holsti, Stephan Thesing, David Whalley, Guillem Bernat, Christian Ferdinand, and Reinhold Heckmann. The worst-case execution-time problem-overview of methods and survey of tools. $A C M$ Trans. on Embedded Computing Systems, 7(3):36:1-36:53, apr 2008.

57. Christian Ferdinand. Worst Case Execution Time Prediction by Static Program Analysis. In Int. Parallel and Distributed Processing Symposium (IPDPS), Santa Fe, NM, USA, 2004. 
58. Jeffery Hansen, Scott Hissam, and Gabriel Moreno. Statistical-Based WCET Estimation and Validation. In Int. Workshop on Worst-Case Execution Time Analysis (WCET), Dublin, Ireland, 2009.

59. Liliana Cucu-Grosjean, Luca Santinelli, Michael Houston, Code Lo, Tullio Vardanega, Leonidas Kosmidis, Jaume Abella, Enrico Mezzetti, Eduardo Quiñones, and Francisco Cazorla. Measurement-based probabilistic timing analysis for multi-path programs. In Euromicro Conf. on Real-Time Systems (ECRTS), Pisa, Italy, 2012.

60. Aloysius Mok and Deji Chen. A multiframe model for real-time tasks. IEEE Trans. on Software Engineering, 23(10), 1997.

61. Noel Tchidjo Moyo, Eric Nicollet, Frederic Lafaye, and Christophe Moy. On schedulability analysis of non-cyclic generalized multiframe tasks. In Euromicro Conf. on Real-Time Systems (ECRTS), Brussels, Belgium, 2010.

62. Martin Stigge, Pontus Ekberg, Nan Guan, and Wang Yi. The digraph real-time task model. In Real-Time and Embedded Technology and Applications Symposium (RTAS), Chicago, IL, USA, 2011.

63. Haibo Zeng and Marco Di Natale. Schedulability Analysis of Periodic Tasks Implementing Synchronous Finite State Machines. 2012 24th Euromicro Conf. on Real-Time Systems, jul 2012.

64. Sanjoy Baruah, Louis E Rosier, and Rodney R Howell. Algorithms and complexity concerning the preemptive scheduling of periodic, real-time tasks on one processor. RealTime Systems, 2, 1990 .

65. Mathieu Desnoyers and Michel Dagenais. The LTTng tracer: A Low Impact Performance and Behavior Monitoring for GNU/Linux. In Linux Symposium, Ottawa, Canada, 2006.

66. David Doose, Christophe Grand, and Charles Lesire. MAUVE Runtime: a componentbased middleware to reconfigure software architectures in real-time. Journal of Software Engineering for Robotics (JOSER), 8(1):128-140, 2017. 